\title{
An Update on Acute Pancreatitis
}

\author{
Catalin V. Ivan ${ }^{1}$ \\ ${ }^{1}$ Gastrointestinal Imaging Group, Department of Radiology, \\ University Hospitals of Leicester, Leicester General Hospital, \\ Leicester, United Kingdom \\ 2Department of Radiology, University of Cincinnati Medical Center, \\ Cincinnati, Ohio United States \\ ${ }^{3}$ Department of Radiology, Mayo College of Medicine Phoenix, \\ Arizona, United States
}

J Gastrointestinal Abdominal Radiol ISGAR 2020;3:14-20
Kumaresan Sandrasegaran²,3 Arumugam Rajesh ${ }^{1}$

\begin{abstract}
Address for correspondence Catalin V. Ivan, Gastrointestinal Imaging Group, Department of Radiology, University Hospitals of Leicester, Leicester General Hospital, Leicester LE5 4PW, United Kingdom (e-mail: catalin.ivan@uhl-tr.nhs.uk).
\end{abstract}

\begin{abstract}
Keywords

- acute pancreatitis

- Atlanta classification

- peripancreatic collections

In recent years, there have been further advances in understanding of the acute pancreatic inflammation and its complications. Among these are emerging concepts such as peripancreatic necrosis and main duct injury secondary to necrotizing pancreatitis. To remain relevant to the clinical teams, radiologists need to be aware of these advances in knowledge and their reports should provide key points of information that may impact patient management. This article provides an up to date review of acute pancreatitis.
\end{abstract}

\section{Introduction}

Acute pancreatitis represents a spectrum of inflammatory pathology of the pancreas, being a common cause of acute gastrointestinal-related surgical admission. ${ }^{1-5}$ The overall mortality is around 6\% varying between 1 and $2 \%$ in mild cases and increasing up to $30 \%$ in severe cases associated with persistent organ failure (beyond 48 hours) and local complications. ${ }^{1-3}$ Gallstones and alcohol are the main causes of acute pancreatitis. However, in up to a third of cases a cause cannot be identified. ${ }^{13}$ At least two of the following criteria need to be fulfilled for the diagnosis of acute pancreatitis: acute onset epigastric pain, at least three times increase in the level of serum amylase or typical computed tomography (CT) findings. ${ }^{4}$ Acute pancreatitis is classified as interstitial or necrotizing pancreatitis (NP), the former representing the majority of such cases $(\sim 90 \%) .^{1-5}$

Acute interstitial pancreatitis (IP) is a mild form of inflammation, confined to the organ and the surrounding fat that typically resolves in less than 4 weeks. ${ }^{3}$ On the other hand, NP accounts for a more complex inflammatory response that can be viewed in stages: an early phase of pancreatitis (lasting for $\sim 1$ week) characterized by the cytokine inflammatory cascade that may lead to systemic inflammatory response syndrome (SIRS) and a later phase. Clinically, SIRS is characterized by at least two of the following: a heart rate of more than 90 beats/min, a respiratory rate of more than $20 / \mathrm{min}$, body temperature of less than $36^{\circ} \mathrm{C}$ or more than $38^{\circ} \mathrm{C}$, a white cell count of less than 4 or greater than $12 \times 10^{9} / \mathrm{L}$, and a $\mathrm{PCO}_{2}$ of less than $32 \mathrm{~mm} \mathrm{Hg}{ }^{2-5}$ SIRS may lead to multiorgan disfunction (especially affecting the kidneys and the lungs). The treatment of patients during this phase is guided by clinical parameters. The role of $\mathrm{CT}$ is limited during the early phase. Imaging is a relatively insensitive predictor of pancreatic necrosis in early stages and imaging findings do not generally reflect the clinical severity. ${ }^{2-4}$ The persistence of SIRS and the development of local complications define the late phase of acute pancreatitis. During this late phase, imaging bears an important role in identifying and characterizing complications (related to the pancreas and the surrounding tissues and vessels)..$^{2-4}$

\section{Imaging Protocol in Acute Pancreatitis}

Although the diagnosis of pancreatitis is based on clinical assessment, contrast-enhanced CT (CECT) is the investigation of choice during later stages in assessing for local complications and helping to exclude malignancy in patients older than 40 years of age with no obvious etiology. ${ }^{4}$ Typically, a parenchymal phase is performed (this is a late arterial phase at 40 second after intravenous contrast administration) to assess for pancreatic necrosis or splenic artery pseudoaneurysm formation and a portal venous phase (at 70 seconds) to depict potential portomesenteric system thromboses or enhancing collections. The rate of the intravenous contrast should typically be 3 to $5 \mathrm{~mL} / \mathrm{s}$ and oral contrast should 
not be routinely administered. Follow-up scans are usually performed in the portal venous phase unless an arterial pseudoaneurysm is being considered in which case an arterial phase should additionally be performed.

\section{Imaging Findings in Acute Interstitial Pancreatitis}

In mild forms of acute IP, there is organ swelling and edema with peripancreatic fat stranding on $\mathrm{CT}$ and magnetic resonance imaging (MRI) (-Fig. 1). The pancreas enhances homogenously post-contrast administration on both modalities. MRI is more sensitive in identifying pancreatic edema than CECT (reflected by organ enlargement and hyperintense pancreatic parenchyma on T2-weighted images). The T1 and fat-saturated T2 sequences are most sensitive in depicting peripancreatic fat stranding. ${ }^{2}$ However, MRI is not routinely performed in acute pancreatitis unless a common bile duct stone (not seen on ultrasound) is suspected in which case a magnetic resonance cholangiopancreatography (MRCP) may be indicated. In more severe forms of IP, there may be formation of peripancreatic collections as fluid continues to leak from the parenchyma.

\section{Imaging Findings Necrotizing Pancreatitis}

The presence of necrosis in the pancreas and/or in the surrounding tissues falls under the umbrella term of NP. Most commonly, there is an association of both pancreatic and peripancreatic necrosis $(75-80 \%) .{ }^{6}$ However, in $20 \%$ of cases peripancreatic only necrosis can be seen, leaving a small minority of cases with organ limited necrosis. ${ }^{6}$

Although historically this has been considered a severe form of disease per se, there is evidence in the medical literature that the mortality in such cases without organ damage or infected collections is similar to those of IP. ${ }^{6}$

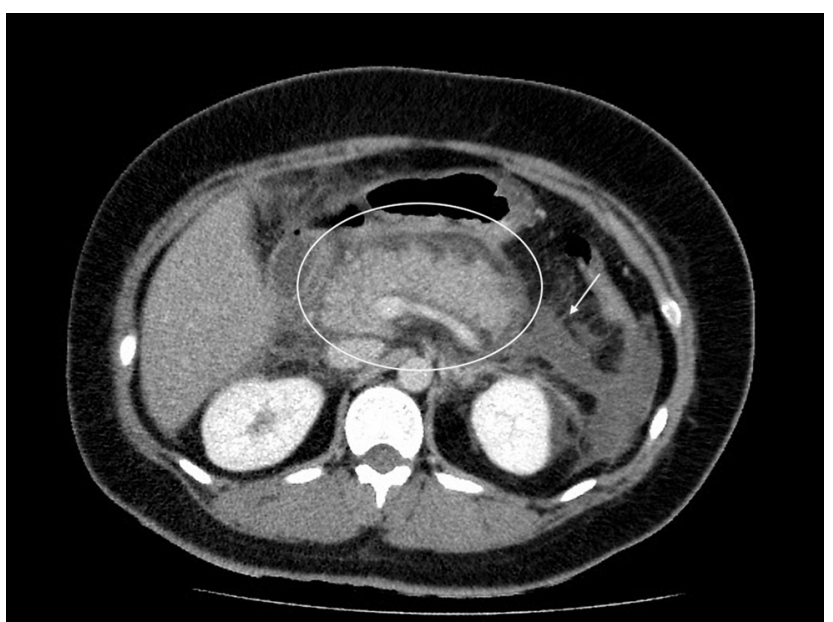

Fig. 1 Acute interstitial pancreatitis with acute peripancreatic fluid collection (APFC). A 55-year-old female patient with a history of gallstones presented with severe epigastric pain and an amylase of $558 \mathrm{U}$ |L. Axial selected image of the portal venous phase of contrast-enhanced computed tomography at 8 days post admission reveals an edematous, swollen pancreas with surrounding retroperitoneal fat stranding (oval). No parenchymal pancreatic necrosis. There is extraparenchymal peripancreatic simple fluid in keeping with acute peripancreatic fluid collections (APFC-arrow).
On CECT, the areas of parenchymal necrosis will not enhance and will appear as one or several areas of parenchymal hypoattenuation (-Fig. 2). The normal enhancing pancreatic parenchyma will measure between 80 and 150 Hounsfield Units (HU), whereas necrotic pancreatic tissue will measure $<30 \mathrm{HU}$. Glandular necrosis can be classified as none, less than $30 \%, 30$ to $50 \%$, or more $50 \%$ of the total pancreas. More recent CT-based classifications (e.g., the CT severity index) regard the extent of necrosis as none, less than $30 \%$, or more than $30 \%$. This will be further discussed later on in the article. Grossly, the head of the pancreas accounts for $\sim 50 \%$ of the gland and the body and tail each accounting for $\sim 25 \%$.The extent of parenchymal necrosis has been shown to correlate with the development of organ failure (high likelihood if the areas of necrosis account for $>30 \%$ of the parenchyma). ${ }^{6}$

It is important that both clinicians and radiologists are aware that the development of pancreatic necrosis will require at least 48 hours and imaging in the first 12 hours may be falsely reassuring. Ideally, the CT scan should be delayed at least 3 days since the initial diagnosis to ensure good sensitivity in pancreatic necrosis detection ( $<70 \%$ sensitivity within the first 72 hours) $)^{2,6}$

\section{Imaging of Collections in Pancreatitis}

The correct nomenclature of fluid collections associated with IP under the revised Atlanta classification is acute peripancreatic fluid collections (APFC) if they form in the first 4 weeks after the onset of symptoms or pseudocysts if they persist beyond this time frame ${ }^{1-6}(-$ Fig. 3 ).

The development of APFC is not uncommon (seen in up to $40 \%$ of cases) and is generally formed in the first 48 hours

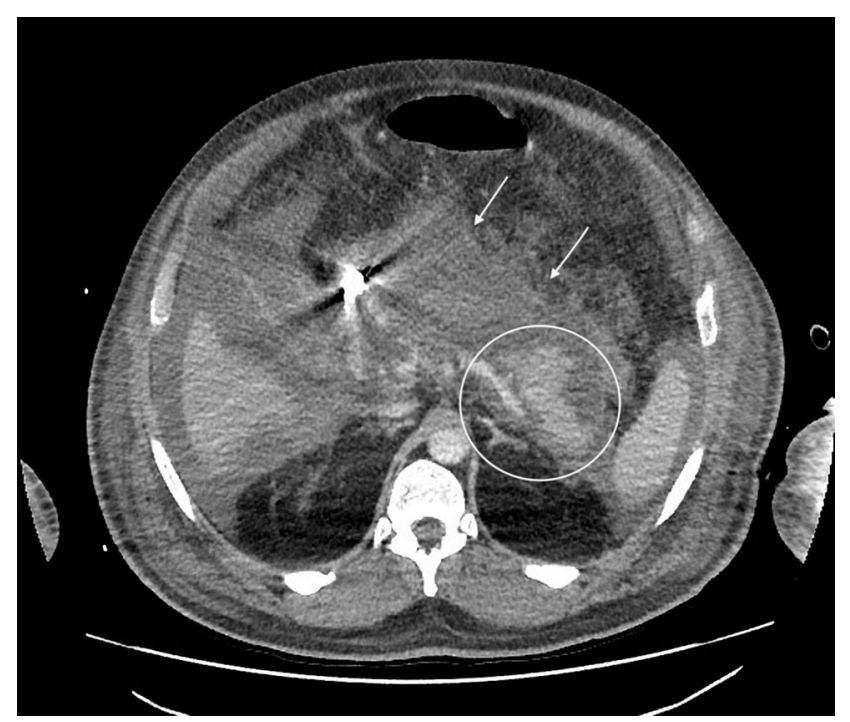

Fig. 2 Acute necrotizing pancreatitis. A 67-year-old male patient with a history of alcohol intake presented with epigastric pain and raised amylase. Axial selected image of the portal phase computed tomography scan at 5 days post admission shows $>50 \%$ pancreatic parenchymal necrosis (hypoattenuating, nonenhancing parenchyma) sparing the tail (circle). There is fluid within and around the pancreas (arrows) in keeping with acute intra and extraparenchymal necrotic collections tracking intraperitoneal around the liver and splenic capsule. 


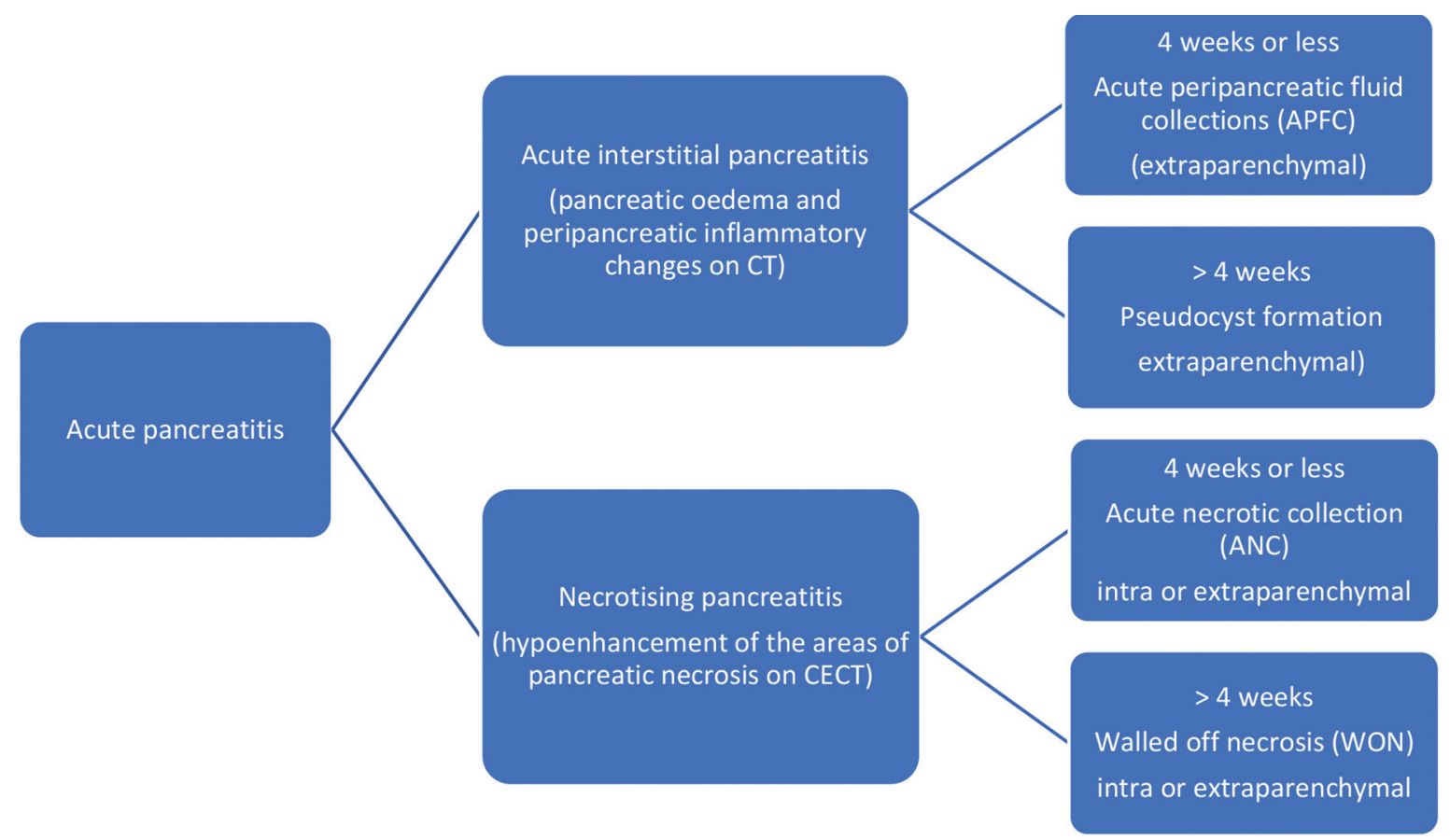

Fig. 3 Flowchart - Classification and correct nomenclature of the collections associated with acute pancreatitis. CECT, contrast-enhanced computed tomography.

(-Fig. 1). ${ }^{6}$ They develop around the pancreas but can potentially track in the pelvis or mediastinum. On CT, these demonstrate fluid density and lack of a wall. APFC only requires drainage if they become infected (considering the iatrogenic risk of a sterile infection becoming infected). Imaging signs that these collections may have become infected are the presence of gas and peripheral enhancement. ${ }^{6,7}$ Forty to fifty percent of these collections resolve spontaneously, while the rest persist beyond 4 weeks and become encapsulated by a fibrous wall, forming a pseudocyst. ${ }^{2,6}$

Pseudocysts smaller than $4 \mathrm{~cm}$ are likely to resolve spontaneously, whereas those larger than $10 \mathrm{~cm}$ require drainage. Drainage may be performed percutaneously via CT and ultrasound or endoscopically via a transgastric or transduodenal approach or surgical depending on the access. An endoscopic transpapillary approach to drainage may be considered in cases of pancreatic ductal injury communicating with the pseudocyst. In such cases, MRCP or endoscopic pancreatic ductography should be considered prior to the procedure to assess the integrity of the pancreatic duct. Pseudocyst rupture, although a rare complication, may be associated with hemorrhagic shock and high mortality of up to $40 \%{ }^{6}$ (-Fig. 4). Moreover, large pseudocysts can have mass effect leading to gastric outlet obstruction, may fistulize into the gastrointestinal tract, or erode into nearby vessels. ${ }^{2}$

The collections associated with NP during the first 4 weeks of symptom onset are called acute necrotic collections (ANC- - Fig. 2).$^{1-6}$ On CT, these appear similar to the APFC during the first week but may become more heterogeneous in the following weeks (due to necrotic debris content). Further features differentiating ANC from APFC include possible intrapancreatic location, may be multiple or multiloculated and can form in various anatomical locations. Beyond the 4 weeks' time frame, these are referred as walled off pancreatic necrosis (WON- - Fig. 5). ${ }^{1-6}$

WONs appear as encapsulated collections of fluid in the region of previous necrotic tissue. Although it can be mistaken on CT for a pseudocyst, there are features that differentiate it from the later: larger size with possible extension laterally into the paracolic gutters or inferiorly down the root of mesentery, thick or multiple septations, fat debris, irregular wall, and pancreatic parenchymal deformity.1,2, Collections in close proximity to the lesser sac can be drained endoscopically through the gastric wall ( - Fig. 6), whereas those extending in the retrocolic regions are more likely to require surgical intervention.

Infection is more likely to occur in ANCs and WONs than in APFCs and pseudocysts. Gas within a collection is a reliable indicator of infective changes ( - Fig. 7 ). ${ }^{7}$ The correct term when infection occurs is infected- ANC, -WON, -APFC or pseudocyst. Terms such as "pancreatic abscess" are no longer in use. ${ }^{1}$ Similar to ANC, WON can form within the pancreatic parenchyma.

\section{Pancreatic Duct Injury}

Also named disconnected pancreatic duct syndrome is a consequence of main pancreatic duct (MPD) inflammatory injury. ${ }^{2}$ It occurs in up to a third of the NP cases, especially those associated with central necrosis at the pancreatic neck 

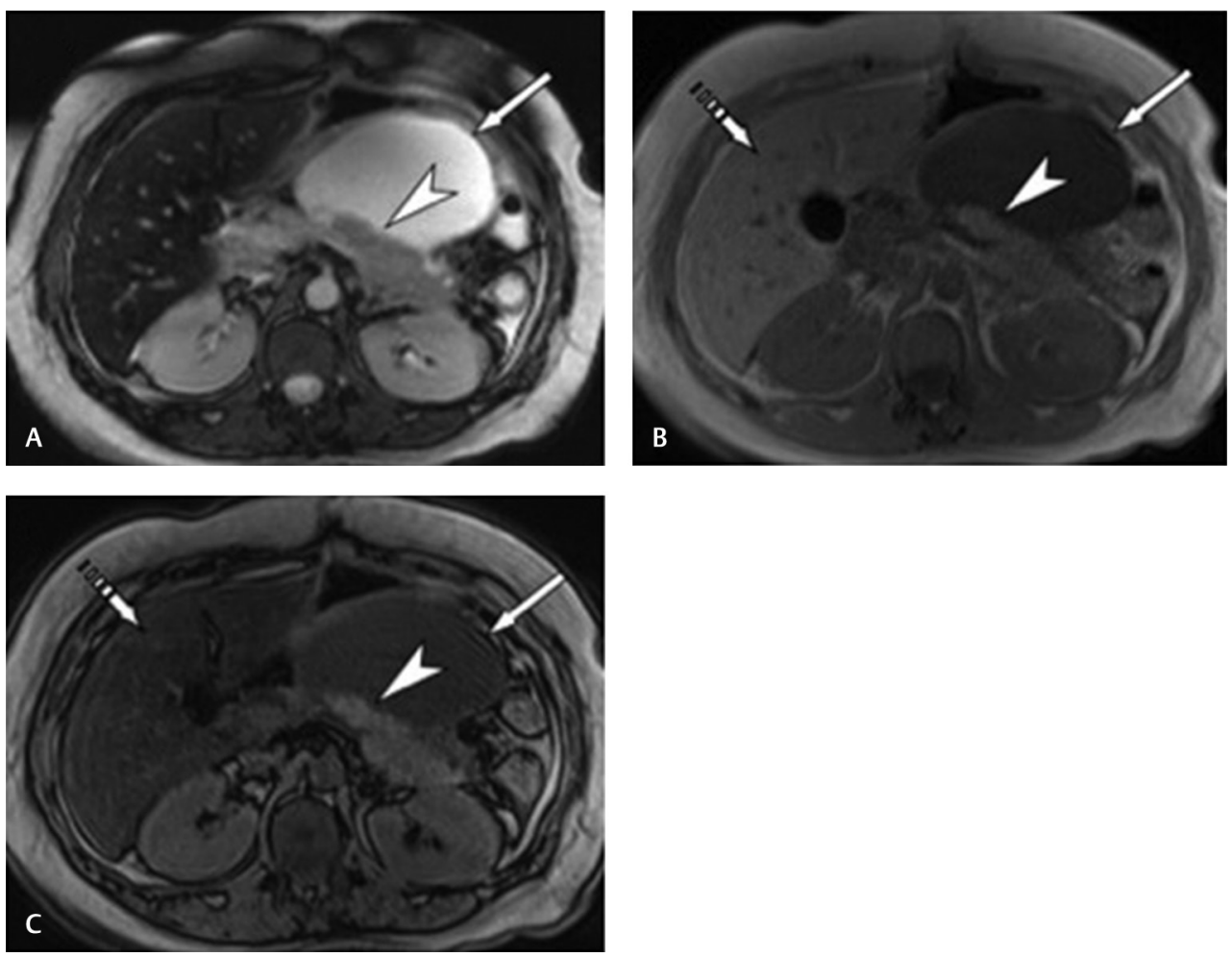

Fig. 4 Hemorrhagic pseudocyst. A 40-year-old female with acute pancreatitis and dropping hemoglobin. (A) Axial T2-weighted image shows a large fluid collection (arrow) with low signal dependent material (arrowhead) (B, C). Axial T1-weighted in-phase (B) and out of phase (C) images show high signal dependent material (arrowhead) in the fluid collection (arrow) indicating blood products in keeping with a hemorrhagic pseudocyst. In addition, there is reduced liver signal (dashed arrows) on opposed-phase image (C) compared with in-phase image, indicating hepatic steatosis.

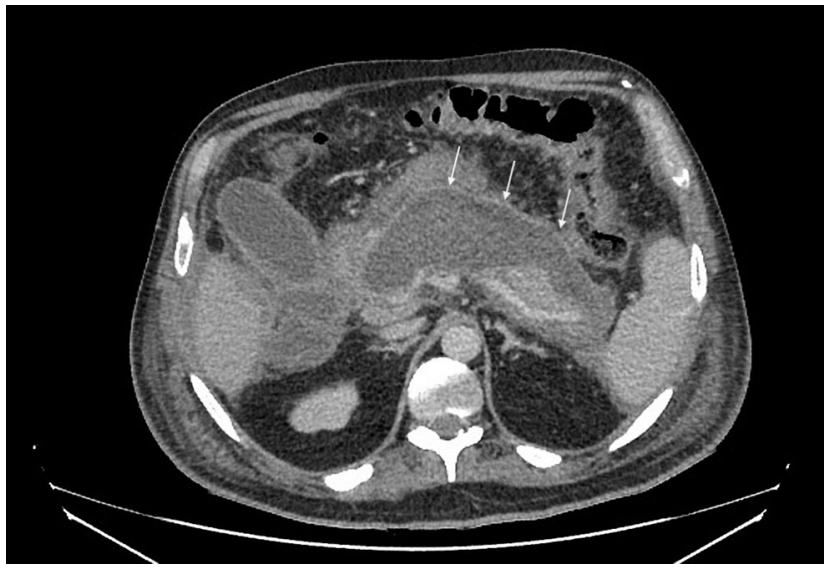

Fig. 5 Acute necrotizing pancreatitis with walled off necrosis (WON). A 62-year-old male presented with severe acute pancreatitis and systemic inflammatory response syndrome and was admitted to the intensive care unit. The inflammatory marker remained persistently high and a follow-up computed tomography scan at 4.5 weeks was organized. This revealed a peripherally enhancing peripancreatic collection in the context of necrotizing pancreatitis (arrows) in keeping with WON.

(anatomically a watershed region). ${ }^{2}$ This leads to the formation of recurrent peripancreatic necrosis and fluid collections that require permanent drainage (endoscopic or surgical) or distal pancreatectomy. ${ }^{8}$ MRCP and endoscopic pancreatic ductography may help in confirming the diagnosis. The former is less invasive and its sensitivity can be enhanced by administering secretin. ${ }^{2}$ MRCP may reveal MPD interruption or a collection of fluid within the region of the duct, communicating with it (-Fig. 8). In cases that remain inconclusive, endoscopic assessment may be considered (representing the gold standard in pancreatic duct assessment). However, endoscopic retrograde cholangiopancreatography is invasive and puts the patient at risk of worsening or recurrent pancreatitis.

\section{Vascular Complications}

The most common vascular complication in acute pancreatitis is venous thrombosis (most frequently affecting the splenic vein but it can involve all of the portomesenteric branches). On CT, this can be depicted as filling defects with possible expansion of the involved veins ( - Fig. 9 ).

Pseudoaneurysm formation is the next most common vascular complication. As the name suggests, these are "false aneurysms" rather than true aneurysms (-Fig. 10). There reflects a defect in the arterial wall secondary to prolonged perivascular inflammation and enzyme leakage. The sac is contained by surrounding connective tissue that makes them prone to bleeding. They develop in later stages as a complication of pseudocysts and WON and are associated with a 9 in 10 mortality rates if left untreated. ${ }^{2}$ The main vessels affected are the splenic, gastroduodenal, and pancreatoduodenal arteries. 

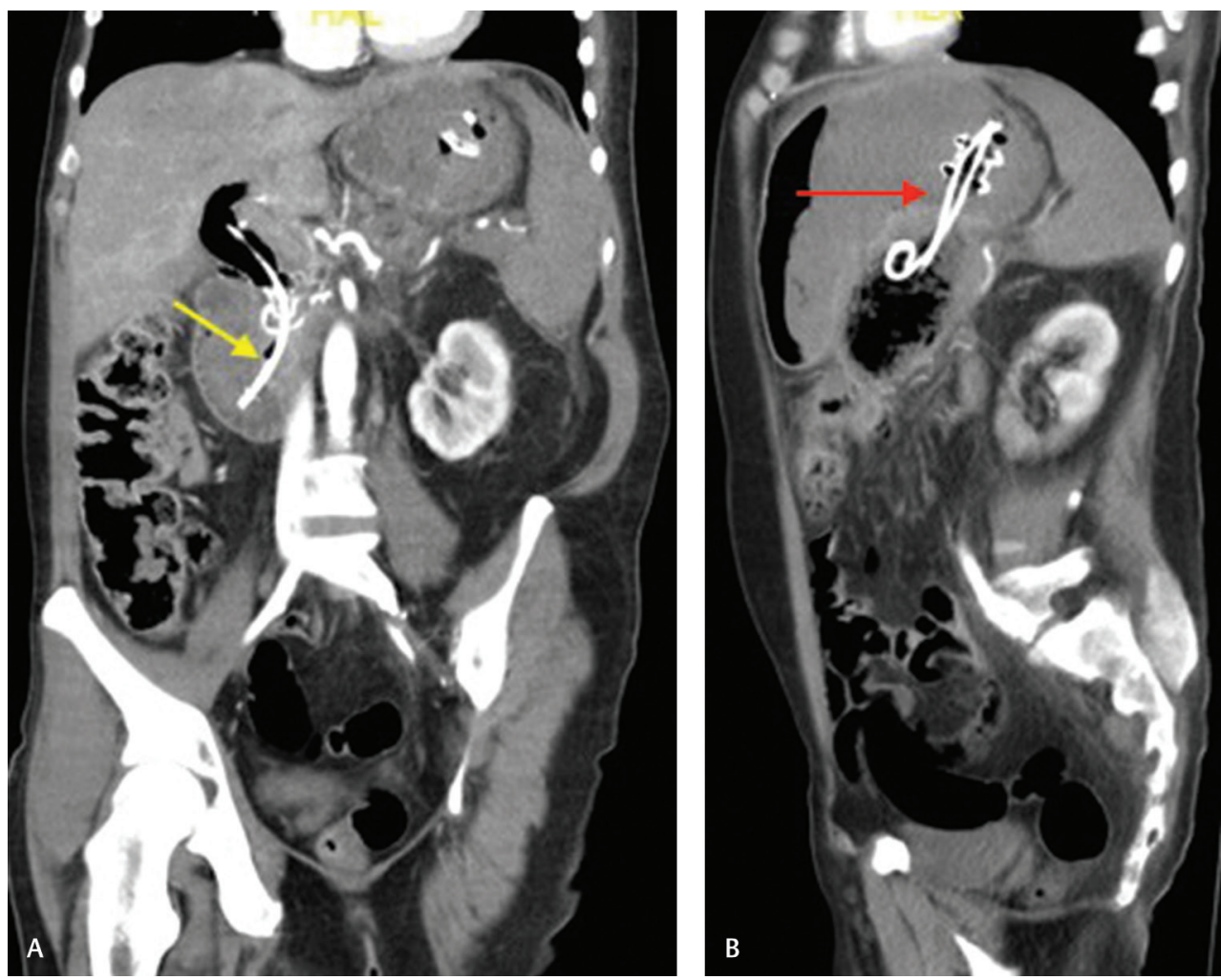

Fig. 6 A 55-year-old man with pancreatitis secondary to choledocholithiasis. (A) Coronal computed tomography showing an internal biliary drain and pneumobilia (yellow arrow). (B) There is an endoscopic ultrasound-guided transmural gastric drainage of the walled off necrotic lesion (red arrow).

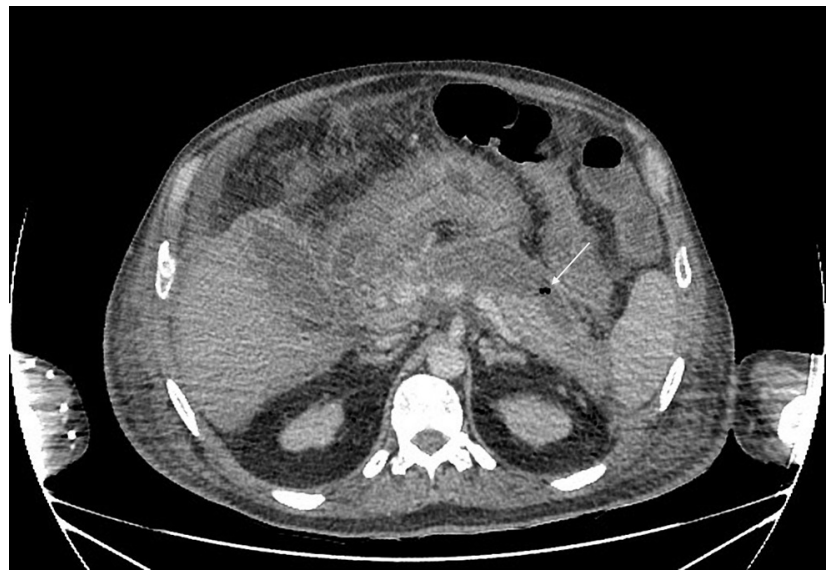

Fig. 7 Acute necrotizing pancreatitis with infected walled off necrosis. Follow-up computed tomography (CT) scan at 6 weeks of the same patient in - Fig. 5. The patient had ongoing swinging fevers and a new CT was requested. On the selected axial CT in portal venous phase, there is persistence of the peripancreatic peripherally enhancing fluid collection which. There are several loculi of gas within this collection (arrow), in keeping with infection).

\section{Radiological Grading System on CT}

The CT severity index was developed in the nineties by Balthazar et $\mathrm{al}^{10}$ to help radiologists provide a consensual grading when reporting scans with acute pancreatitis. Most recently, Mortele et al have proposed a modified, somewhat simplified CT severity index that correlates more closely to the patient outcome than its original. ${ }^{9}$ This represents a
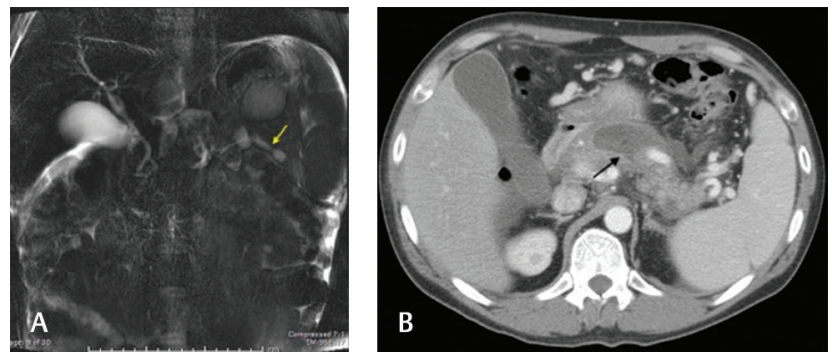

Fig. 8 Pancreatic duct injury. Pancreatic duct injury in two different patients with necrotizing pancreatitis. (A) Coronal thick secretin enhanced magnetic resonance cholangiopancreatography slab shows abrupt cutoff of the pancreatic duct at the level of the tail pf the pancreas (yellow arrow). The pancreatic duct injury at this level is discharging into the peripancreatic collections ( 3 high signal collections seen along the pancreatic duct). (B) Axial portal venous phase computed tomography scan reveals a break into the wall of the dilated pancreatic duct (black arrow), continuing in to a peripancreatic collection.

score base system that takes into account the pancreatic changes and the extent of the parenchymal necrosis along with the extra-pancreatic findings (-Table 1 ). The final score helps assess the severity of acute pancreatitis as following: mild (0-2), moderate (4-6), or severe (8-10).

\section{Conclusion}

In this article, we present an up to date review of acute pancreatitis from the perspective of the reporting radiologist. We highlight the imaging role in diagnosing this condition with its associated complications and the correct 

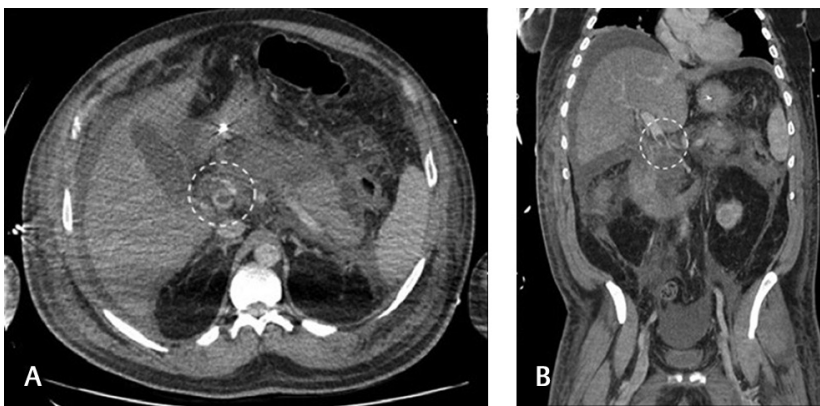

Fig. 9 Portal vein thrombosis. (A, B) Axial and coronal computed tomography (CT). A 55-year--old male presented with acute necrotic pancreatitis after an alcohol binge in the PUB. On the portal venous phase of CT scan performed at day 4 after the acute onset, there is necrosis of the head and neck of the pancreas with acute peripancreatic necrotic collections. There is a central filling defect within the portal vein which is expanded (dotted circle).
Table 1 Modified CT severity score index in acute pancreatitis ${ }^{9}$

\begin{tabular}{|l|l|}
\hline \multicolumn{2}{|c|}{ Modified CT severity index } \\
\hline Prognostic indicator & Points \\
\hline Normal pancreas & 0 \\
\hline $\begin{array}{l}\text { Intrinsic pancreatic abnormalities with or without } \\
\text { inflammatory changes in the peripancreatic fat }\end{array}$ & 2 \\
\hline $\begin{array}{l}\text { Pancreatic or peripancreatic fluid collection or peripan- } \\
\text { creatic fluid necrosis }\end{array}$ & 4 \\
\hline No pancreatic necrosis & 0 \\
\hline$<30 \%$ pancreatic necrosis & 2 \\
\hline$>30 \%$ pancreatic necrosis & 4 \\
\hline $\begin{array}{l}\text { Extrapancreatic complications (pleural effusion, } \\
\text { ascites, vascular complications gastrointestinal tract } \\
\text { involvement) }\end{array}$ & 2 \\
\hline
\end{tabular}

Abbreviation: CT, computed tomography.
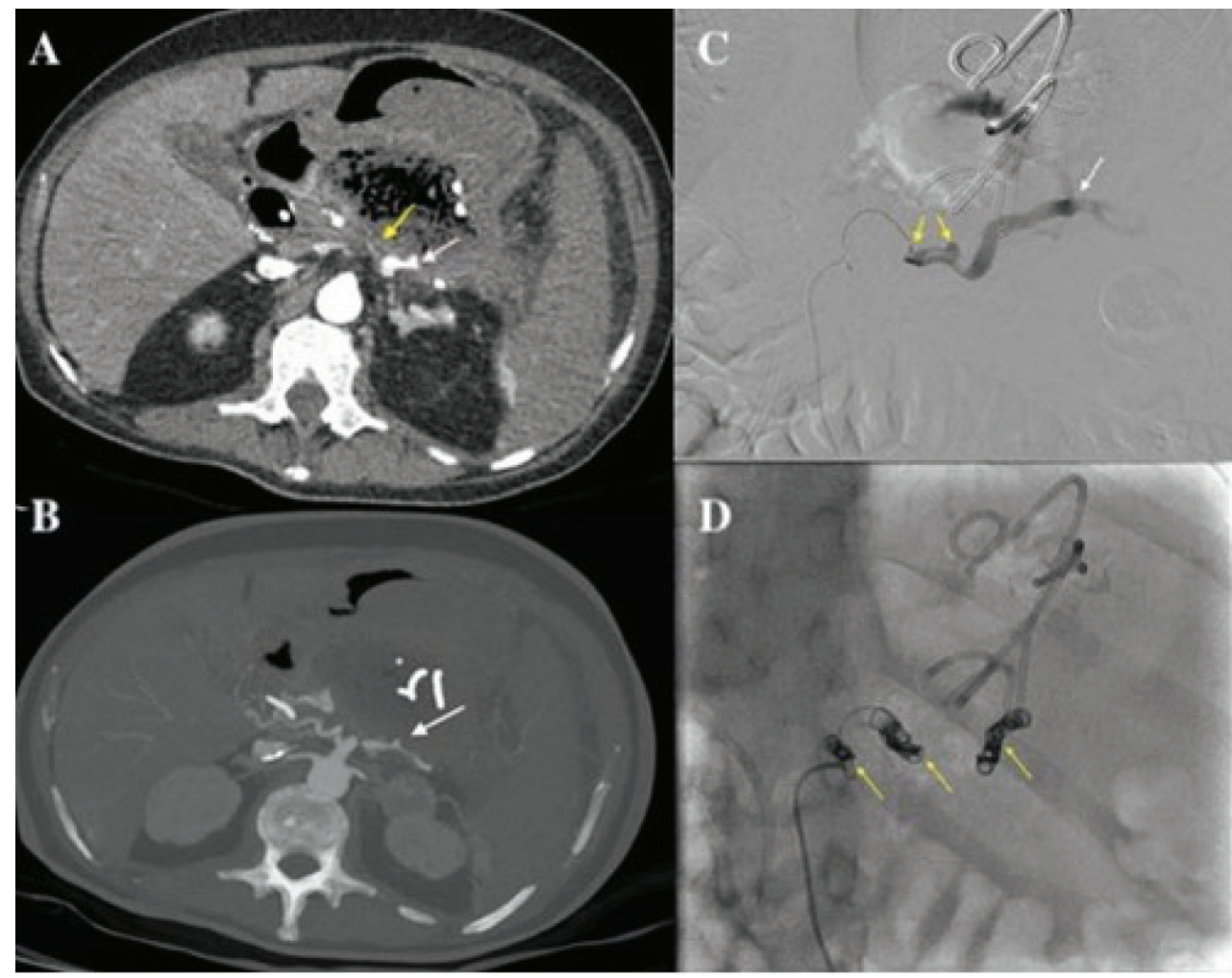

Fig. 10 Splenic artery pseudoaneurysm. A 44-year-old male with necrotizing pancreatitis secondary to choledocholithiasis. (A) Axial computed tomography shows high density material (yellow arrow) in walled-off necrosis. Careful review of major arteries shows swelling of splenic artery (white arrow). Pseudoaneurysm was suspected. (B) Maximum intensity projection image of the splenic artery aneurysm (white arrow) (C) Catheter angiogram-the tip of the catheter is within the proximal splenic artery (yellow arrows)-confirms pseudoaneurysm (white arrow). There is extravasated contrast tracking in to the gas containing collection (red arrow). (D) The pseudoaneurysm was subsequently embolized (yellow arrows showing coils in situ).

nomenclature of the radiological findings in line with the Atlanta Classification. The role of radiology has become essential in modern medicine and radiologists need to anticipate the information needed by the clinical teams in managing these patients. Moreover, radiologists can add value not only by confirming the diagnosis but also in assessing the severity of acute pancreatitis and the extent of potential associated complications. Our report can help guide the clinicians in how to best approach these complications especially when nowadays, there is an arsenal of options much less invasive than conventional surgery.

\section{Conflict of Interest}

None declared. 


\section{References}

1 Murphy KP, O'Connor OJ, Maher MM. Updated imaging nomenclature for acute pancreatitis. AJR Am J Roentgenol 2014;203(5):W464-9

2 Zhao K, Adam SZ, Keswani RN, Horowitz JM, Miller FH. Acute pancreatitis: revised Atlanta classification and the role of cross-sectional imaging. AJR Am J Roentgenol 2015;205(1):W32-41

3 Foster BR, Jensen KK, Bakis G, Shaaban AM, Coakley FV. Revised Atlanta classification for acute pancreatitis: a pictorial essay. Radiographics 2016;36(3):675-687

4 Thoeni RF. The revised Atlanta classification of acute pancreatitis: its importance for the radiologist and its effect on treatment. Radiology 2012;262(3):751-764

5 Shyu JY, Sainani NI, Sahni VA, et al. Necrotizing pancreatitis: diagnosis, imaging, and intervention. Radiographics 2014;34(5):1218-1239
6 Zaheer A, Singh VK, Qureshi RO, Fishman EK. The revised Atlanta classification for acute pancreatitis: updates in imaging terminology and guidelines. Abdom Imaging 2013;38(1):125-136

7 Hughey M, Taffel M, Zeman RK, Patel S, Hill MC. The diagnostic challenge of the sequelae of acute pancreatitis on CT imaging: a pictorial essay. Abdom Radiol (NY) 2017;42(4):1199-1209

8 Nadkarni NA, Kotwal V, Sarr MG, Swaroop Vege S. Disconnected pancreatic duct syndrome: endoscopic stent or surgeon's knife? Pancreas 2015;44(1):16-22

9 Mortele KJ, Wiesner W, Intriere L, et al. A modified CT severity index for evaluating acute pancreatitis: improved correlation with patient outcome. AJR Am J Roentgenol 2004;183(5):1261-1265

10 Balthazar EJ, Robinson DL, Megibow AJ, Ranson JHC. Acute pancreatitis: value of CT in establishing prognosis. Radiology 1990;174:331-336 